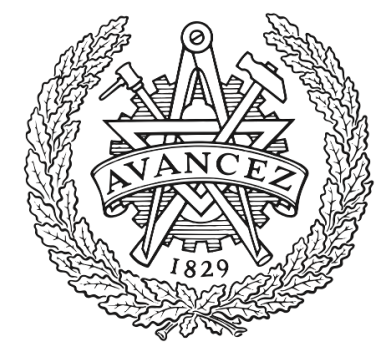

CHALMERS

UNIVERSITY OF TECHNOLOGY

\title{
Optical injection locking at sub nano-watt powers
}

Downloaded from: https://research.chalmers.se, 2023-04-26 12:17 UTC

Citation for the original published paper (version of record):

Kakarla, R., Schröder, J., Andrekson, P. (2018). Optical injection locking at sub nano-watt powers.

Optics Letters, 43(23): 5769-5772. http://dx.doi.org/10.1364/OL.43.005769

N.B. When citing this work, cite the original published paper. 


\title{
Optical injection locking at sub nano-watt powers
}

\author{
Ravikiran Kakarla, ${ }^{*}$ Jochen Schröder, and Peter A. Andrekson \\ Photonics Laboratory, Department of Microelectronics and Nanoscience, Chalmers University of Technology, Gothenburg, 41296, Sweden \\ *Corresponding author: kakarla@chalmers.se
}

Received 28 September 2018; revised 28 October 2018; accepted 29 October 2018; posted 29 October 2018 (Doc. ID 346875$)$; published 26 November 2018

\begin{abstract}
We demonstrate optical injection locking (OIL) at record low injection power of $-65 \mathrm{dBm}$ using EDFA-based preamplification and an electrical phase locked loop (PLL). Investigating the phase noise characteristics of OIL, we find that at low injection powers the slave laser linewidth and injection ratio strongly influence the phase noise of the locked slave output. By introducing an EDFA pre-amplifier, the minimum locking power for OIL is reduced. Moreover, using this pre-amplifier we find that there exists an optimum injection power into the slave where the output phase noise is minimized and is below the phase noise without EDFA. We evaluate an OIL-based pump recovery in a phase sensitive amplifier (PSA) receiver system aimed at free-space communications. () 2018 Optical Society of America
\end{abstract}

https://doi.org/10.1364/OL.43.005769

Optical injection locking (OIL) is a mechanism where a free running slave laser is injected with coherent light from a master laser which causes the slave laser to oscillate at the master's frequency and follow the master's phase. OIL has been widely used in frequency stability improvement of lasers [1,2], coherence control and polarization switching in VCSELs $[3,4]$ and carrier recovery for homodyne receivers $[5,6]$ with reduction of phase noise [7]. Precise phase and frequency restoration of an optical carrier along with a simple implementation makes OIL an interesting technique for carrier recovery. We are particularly interested in OIL for pump recovery in phase sensitive amplifiers (PSA) at very low injection powers. PSAs are known for low noise optical amplification (a noise figure of $1.1 \mathrm{~dB}$ demonstrated [8]) and are therefore promising for applications where high sensitivity reception of optical signals is critical, such as deep space communications. However, PSAs require the presence of a pump wave to fulfill their promise for these high sensitivity applications. The limited power budget requires that the power of the transmitted pump is minimized, which necessitates OIL at the receiver at extremely low powers.

OIL has previously been demonstrated at low injection powers. In Ref. [9], the locking power could be reduced to $-60 \mathrm{dBm}$ [injection ratio (IR), $P_{\text {inj }} / P_{\mathrm{sl}}=-65 \mathrm{~dB}$ ] with the help of a PLL, which improved the locking stability at lower powers. However, to fully take advantage of OIL for high sensitivity PSA a further reduction is necessary. For example, we have shown the PSA sensitivity of $-50.2 \mathrm{dBm}(3.7$ photons per bit) at $10^{-3}$ BER for a $10 \mathrm{GBd}$ QPSK signal by considering only signal and idler powers at the receiver [10]. At such low power the received pump power needs to be less than $-65 \mathrm{dBm}$ to keep the sensitivity penalty below $0.1 \mathrm{~dB}$. In this Letter, we investigate different scenarios of OIL at low input powers with a focus on the phase noise introduced by the OIL. We discuss the implementation of an electrical PLL used to stabilize the OIL, allowing it to operate at injected powers of $-55 \mathrm{dBm}$, corresponding to an IR of $-75 \mathrm{~dB}$. By introducing an EDFA pre-amplifier, we reduce the locking power to $-65 \mathrm{dBm}$ (OSNR of $-11.5 \mathrm{~dB}$ ). We briefly discussed these results previously [11], to which this Letter is an extension. Here we also discuss the prospects of using this OIL setup as a pump regenerator in a PSA-based receiver and quantify the sensitivity penalty due to OIL based pump recovery operating at very low power levels.

One of the key parameters of OIL is the locking bandwidth, which depends on the amount of optical power injected $P_{\text {inj }}$ into the slave laser and is expressed as [12]

$$
\Delta \omega_{\mathrm{LB}}=\sqrt{1+\alpha^{2}} f_{d} \sqrt{\frac{P_{\mathrm{inj}}}{P_{\mathrm{sl}}}},
$$

where $\alpha$ is the linewidth enhancement factor, and $f_{d}$ is the longitudinal mode spacing. Locking bandwidth is the range of frequencies within which both free running frequencies should exist in order to lock. Typical thermal drifts of the slave laser cause frequency drifts up to $100 \mathrm{MHz}$ with respect to the master frequency. In order to lock to these drifts, the locking bandwidth should be higher than the drift bandwidth. The required IR, calculated from Eq. (1), should be higher than approximately $-65 \mathrm{dBm}$. Performing injection locking below these IRs is challenging without any external assistance, such as a PLL. The operation of the PLL in stabilizing the OIL can be understood as follows: in a steady state, the frequency difference between free-running master and slave lasers $\Delta f_{m, \mathrm{sl}}=f_{m}-f_{\mathrm{sl}}$ is related to the phase difference between the incoming master and locked slave output $\Delta \phi_{L}$ by [12]. This can be expressed as

$$
\Delta \phi_{L}=-\sin ^{-1}\left(\frac{\Delta f_{m, \mathrm{sl}}}{\Delta f_{\mathrm{LB}}}\right)-\tan ^{-1} \alpha .
$$

Therefore the PLL, by experimentally obtaining $\Delta \phi_{L}$, can be used to compensate for $\Delta f_{m, \mathrm{sl}}$ and thus keep the OIL in a 
locked state. The implementation of PLL is similar to the work $[9,13]$.

Our experimental OIL setup is shown in Fig. 1. Light from the master laser (NKT photonics, $100 \mathrm{~Hz}$ specified linewidth) is modulated with a $10 \mathrm{GHz}$ signal $\left(f_{0}\right)$ using an amplitude modulator. The modulated tones (center carrier and side tones) are injected into the slave laser (DFB laser, EM4, $50 \mathrm{kHz}$ linewidth, set to $+20 \mathrm{dBm}$ output power) through a circulator, allowing the center carrier tone to lock, $10 \mathrm{GHz}$ side tones are well outside the locking bandwidth without affecting the locking process and are reflected by the slave cavity [9]. The power into the slave $P_{\mathrm{SL}-\text { in }}$ is controlled using a VOA 2. At the slave laser output, the locked carrier wave will have the slave's phase while the side tones will have the master's phase. In contrast to $[9,13]$, here we chose a higher $f_{0}, 10 \mathrm{GHz}$, instead of $1 \mathrm{GHz}$ to make sure the sidebands are completely unaffected by the locking mechanism. The $10 \mathrm{GHz}$ phase difference between master and locked slave is obtained in the photodetector by beating the carrier and side tones and down-converted to a baseband voltage signal with a mixer. The obtained signal then drives the slave laser current to compensate for the relative frequency drift.

The noise performance of the OIL process is measured by beating the slave output with the injected master laser, which was shifted by $27 \mathrm{MHz}$ using an acousto-optic modulator $(\mathrm{AOM})$, in a coherent receiver. The data was digitized at $50 \mathrm{GS} / \mathrm{s}$ over a $40 \mu$ s time window for each batch using a real time scope, then post-processed by performing I-Q imbalance and frequency offset compensation. Longer duration of the time window results in additional phase fluctuations from signal and local oscillator (LO) path vibrations, hence we restricted to it $40 \mu \mathrm{s}$. Amplitude and phase noise standard deviation (std) are quantified from the obtained samples. The amplitude noise std was 0.04 (normalized to mean), and independent of the slave input power in agreement with [14]. Figure 2(a) shows the phase noise std variation with input locking power, with and without PLL. We consider OIL to be stable when it is in locked state a continuous stretch of at least 10 min measurement time. In the experiment, OIL is locked stably more than an hour and gets disturbed by external noise and polarization drifts. The minimum locking power for stable OIL without PLL was $-45 \mathrm{dBm}$ and is reduced to $-55 \mathrm{dBm}$ with the PLL. The corresponding IR is $-75 \mathrm{~dB}$, almost $10 \mathrm{~dB}$

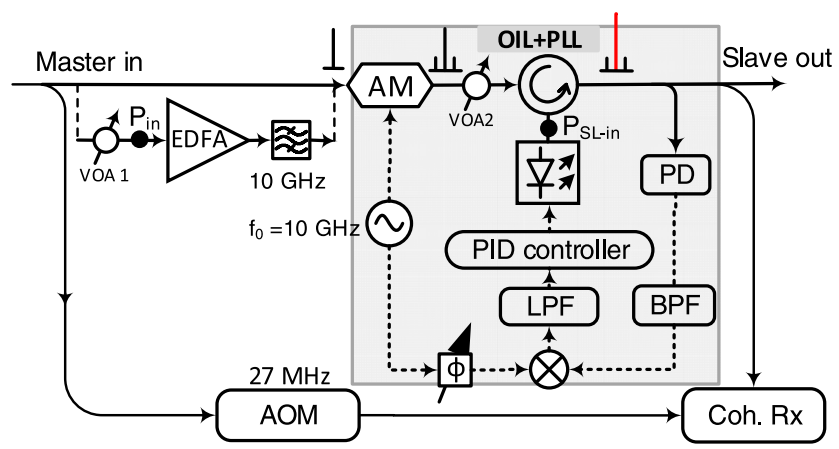

Fig. 1. Setup of optical injection locking using PLL and EDFA preamplifier (EDFA, erbium-doped fiber amplifier); AM, amplitude modulator; PD, photodiode; LPF, low pass filter; BPF, band pass filter; PID, proportional integrator differentiator; VOA; variable optical attenuator.
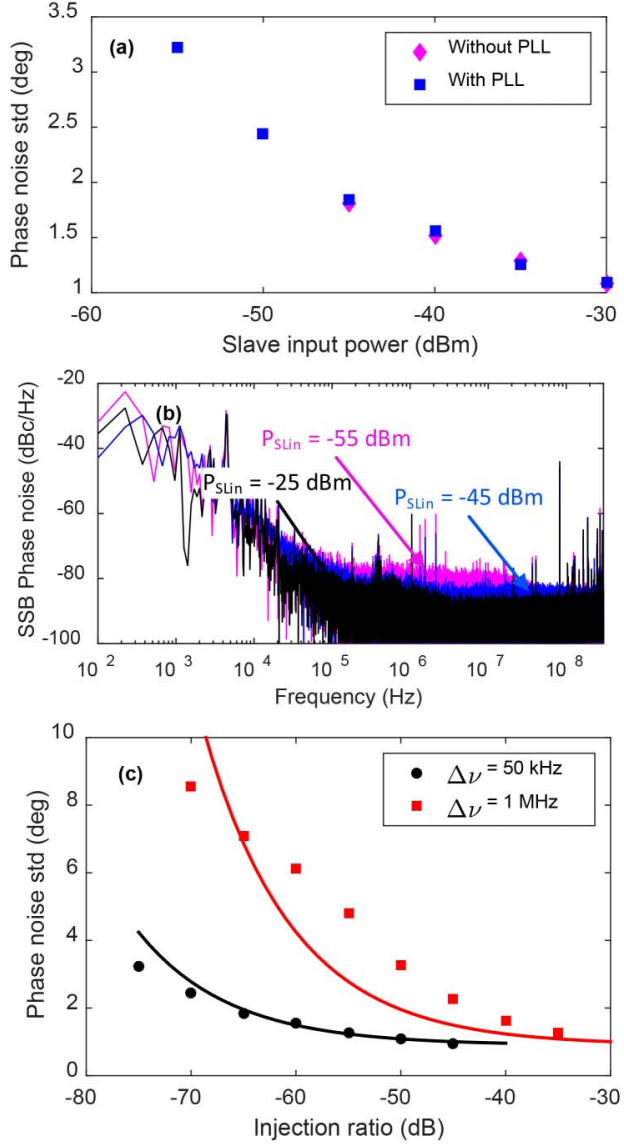

Fig. 2. (a) Comparison of measured locked phase noise of OIL with and without using PLL at different slave input powers; (b) phase noise spectrum for different injection powers; (c) comparison of measured locked phase noise for different slave lasers having linewidths $50 \mathrm{kHz}$ and $1 \mathrm{MHz}$ for different injection ratios. Solid lines are theoretical phase noise curves, and dots are experimental values.

lower than the minimum IR in Ref. [9]. We believe that the improvement is primarily due to high modulation frequency. As in our previous attempts at using a modulation frequency similar to [9], we were not able to achieve stable locking at low IRs. Smaller linewidths of master and slave were also advantageous as smaller frequency drifts allow more stable locking at low IRs [12].

We also see that the phase noise increases with reduced slave input power, to understand this we studied the spectrum of phase noise performing FFT of digital samples measured for different slave input powers shown in Fig. 2(b). From $100 \mathrm{~Hz}$ to $2 \mathrm{kHz}$, phase noise is unstable because of the path vibrations mentioned before. The peak at $83 \mathrm{MHz}$ is due to reflections in circulator port two and the slave laser, however, these do not affect the locking mechanism as the locking bandwidth is less than $40 \mathrm{MHz}$ for $-55 \mathrm{dBm}$ power (IR, $-75 \mathrm{~dB}$ ), and the noise within the locking bandwidth increases with reducing injected power into the slave as shown in Fig. 2(b). This can be understood by observing the steady state in Eq. (2). The locked slave output experiences a phase shift corresponding to the frequency difference $\Delta f_{m, \mathrm{sl}}$. Any frequency drift in master or slave lasers therefore cause phase 
fluctuations in the locked slave output, as discussed in Ref. [9]. However, the source for these frequency variations is not the thermal drifts and current drifts as stipulated in Ref. [9], because in our measurements the phase noise std variation remains mostly unchanged over all the data batches taken at different times. Instead we assume that they originate from the instantaneous frequency variations due to the linewidths of master and slave lasers resulting in phase variations in the locked slave output, which will be enhanced when decreasing the locking bandwidth according to Eq. (2). Since the linewidth of our master laser is very narrow $(100 \mathrm{~Hz})$ compared to the slave linewidth $(50 \mathrm{kHz})$, the phase noise should be dominated by the slave linewidth. To verify this linewidth dependence, we performed a second set of measurements with a $1 \mathrm{MHz}$ linewidth slave laser (DFB laser, Eblana photonics) and compared it against the $50 \mathrm{kHz}$ slave laser as shown in Fig. 2(c). The experimental results are indicated by dots and squares. Clearly, the phase noise of a $1 \mathrm{MHz}$ linewidth laser is higher compared to a $50 \mathrm{kHz}$ laser, indicating that the slave linewidth influences the locked slave output phase noise.

In addition, we performed numerical modeling [lines in Fig. 2(c)] of the phase noise according to Eq. (2), where we consider the free running slave frequencies to be time varying due to their linewidths, calculated as $f_{\mathrm{sl}}(t)=d \phi_{\mathrm{sl}}(t) / d t$. The corresponding time varying phase $\phi_{\mathrm{sl}}(t)$ causing the linewidth is mathematically modelled as a Wiener random process given by

$$
\phi_{\mathrm{sl}}(t)=\phi(0)+\sigma_{\mathrm{PN}} \int_{0}^{t} v(\tau) \mathrm{d} \tau,
$$

where $\sigma_{\mathrm{PN}}$ is the standard deviation, and $v(\tau)$ is the normal distribution function. Ignoring phase noise of the master, Eq. (2) can be written as

$$
\Delta \phi_{L}(t)=-\sin ^{-1}\left(\frac{f_{m}-f_{\mathrm{sl}}(t)}{\Delta f_{\mathrm{LB}}}\right)-\tan ^{-1} \alpha,
$$

where $\alpha$ is used as a fitting parameter for the model, having a value of 7 , and $f_{d}$ is measured experimentally to be $43 \mathrm{GHz}$ for both lasers. The numerical curves closely follow the experimental data supporting our reasoning.

The minimum locking power of OIL is $-55 \mathrm{dBm}$ (IR $=-75 \mathrm{~dB})$ and is achieved using PLL. To further reduce the locking power, we introduce a pre-amplifier to amplify the weak signal before the injection locking. An EDFA with NF = $4.5 \mathrm{~dB}$ operated under constant gain mode, is introduced before the OIL as shown in Fig. 1. A band pass filter after the EDFA is used to remove amplified spontaneous emission (ASE) noise. The ASE present within the locking bandwidth of the slave is transferred to the output signal. Hence, the filter bandwidth is chosen to be as narrow as possible, i.e., $10 \mathrm{GHz}$.

We studied the effect of ASE noise transfer by performing a EDFA input power sweep $P_{\text {in }}$ using VOA1 (indicated in Fig. 1) for different slave input powers $P_{\text {SL-in }}$, varied using VOA2 (indicated in Fig. 1) as shown in Fig. 3(a). Since the EDFA increases the IR by amplifying the low power input, it allows stable locking at further low powers, which is $-65 \mathrm{dBm}$. Since amplitude noise is suppressed by injection locking and it does not change much with OSNR by measurements, it is not discussed. However, the phase noise of the locked signal increases with decrease of $P_{\text {in }}$ value from $-50 \mathrm{dBm}$ to $-65 \mathrm{dBm}$ (OSNR, 3.5 to $-11.5 \mathrm{~dB}$ ) for different $P_{\mathrm{SL}-\text { in }}$ values because of the

\section{Pump OSNR}
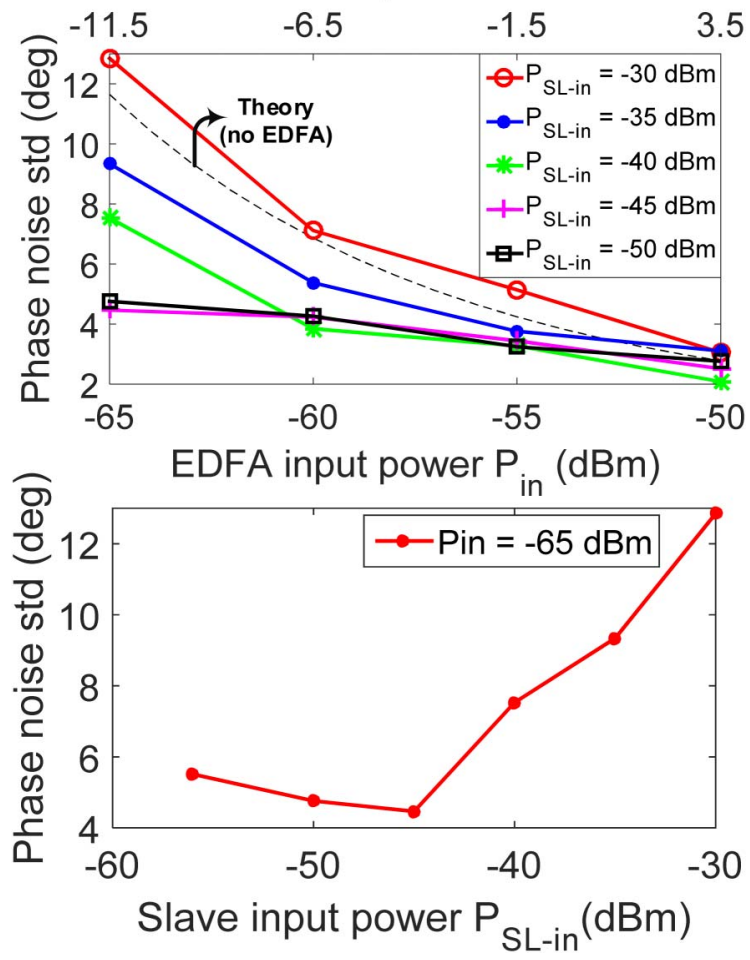

Fig. 3. (a) Measured phase noise variation with EDFA input power (bottom $\mathrm{x}$-axis) or OSNR (top x-axis) for different slave input powers; (b) measured phase noise varying the slave input power for a fixed EDFA input power.

increase in ASE noise within the locking bandwidth. The amount of phase noise transferred depends on the $P_{S L-i n}$ injected into the slave. Hence it is required to minimize $P_{\text {SL-in }}$ without affecting the stability.

To investigate the limit of slave input power $P_{\text {SL-in }}$ for stable locking, we decreased the $P_{\text {SL-in }}$ into the slave using VOA 2 for a fixed $P_{\text {in }}$ value- $65 \mathrm{dBm}$ as shown in Fig. 3(b). The expected behavior is that phase noise will decrease with $P_{\text {SL-in }}$ as the ASE level decreases. But we observe two different behaviors in Fig. 3(b). When $P_{\text {SL-in }}$ reduced from -30 to $-45 \mathrm{dBm}$, initially the phase noise decreases due to ASE transfer decrease as the locking bandwidth decreases. But from $-45 \mathrm{dBm}$ to $-55 \mathrm{dBm}$, phase noise increases again, due to the slave laser linewidth causing phase noise increase with reducing IR.

Therefore, there exists a slave input power for which phase noise transfer can be the minimum. The minimum value of phase noise is obtained in our case at $P_{\text {SL-in }}$ of $-45 \mathrm{dBm}$ and is same for all the $P_{\text {in }}$ values. In Fig. 3(a), the pink line shows the minimum phase noise std at $P_{\text {SL-in }}(-45 \mathrm{dBm})$ for all $P_{\text {in }}$ values, which is less than five deg. The dashed black line shows the theoretical phase noise of OIL when no-EDFA is used, and this is much higher compared to a with-EDFA case at optimized $P_{\text {SL-in }}$. This means, phase noise generated with an EDFA pre-amplifier can be much lower than without it. The optimized phase noise std measured at input power $-65 \mathrm{dBm}$ is $5 \mathrm{deg}$, which is much less compared to the phase noise std measured in Ref. [9], 15 deg at $-60 \mathrm{dBm}$ input power. In conclusion, EDFA not only allows it to operate at 

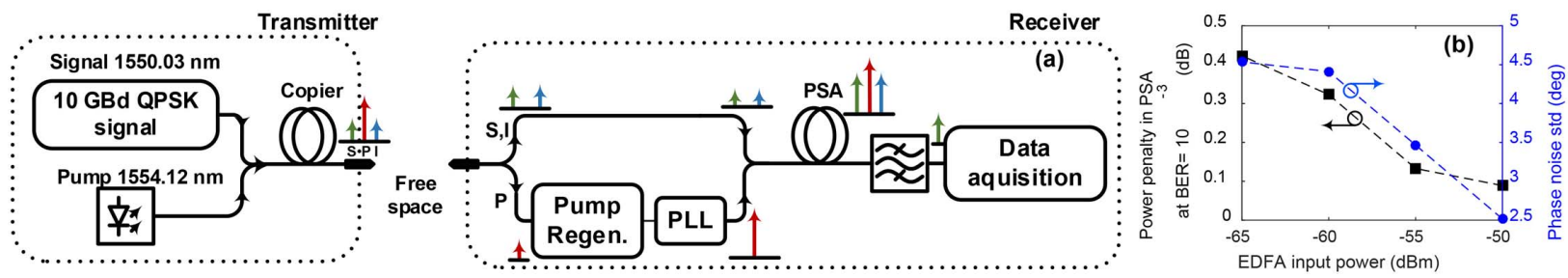

Fig. 4. (a) Setup for PSA based transceiver. S, I, P-signal, idler, and pump waves; (b) measured sensitivity degradation (left y-axis) due to the phase noise (right y-axis) generated in OIL for different pre-amplifier input powers.

much lower power but also can reduce the amount of phase noise transferred.

We used the OIL set up as a pump recovery stage for a PSAbased receiver system. In the setup in Fig. 4(a), the transmitter stage consists of a copier where a signal and pump are combined in a HNLF to generate an idler wave. The three waves are transmitted through the free-space channel. At the receiver stage, the pump is separated from the signal-idler path in order to undergo regeneration where pre-amplifier OIL is performed. All the three waves are then combined for PSA in the HNLF stage. Another PLL is used in the pump regenerator path, to maintain the relative phase of the pump with respect to the signal-idler path constant to achieve maximum PSA gain. The amplified signal after PSA is filtered and digitized using a sampling oscilloscope. The pump regeneration is performed at $-65 \mathrm{dBm}$ input power. The phase noise generated in the pump causes fluctuations in the phase-sensitive gain. The PLL used to achieve maximum PSA gain cannot compensate for these phase fluctuations due to its slower phase tracking capability. The output signal power of a PSA [15] under an ideal case of perfect phase matching is

$$
P_{s}=P_{s 0}\left[\cosh \left(2 g_{0} L\right)+\sinh \left(2 g_{0} L\right) \sin \left(\phi_{s}+\phi_{i}\right)\right],
$$

where the gain coefficient $g_{0}=\gamma P_{P}$ with $P_{P}$ denoting the pump power into the PSA, $L$ and $\gamma$ are the length and nonlinear coefficient of the nonlinear medium, and $P_{s 0}$ is the input signal power. $\phi_{s}+\phi_{i}$ is the relative phase of the signal and idler with respect to the pump. From Eq. (5) the pump phase noise would affect the PSA gain and degrade the signal quality. In Fig. 4(b) we quantified the degradation of the signal quality as a power penalty at $\mathrm{BER}=10^{-3}$ with respect to the ideal case where noOIL was used, and the pump after copier is directly utilized in the PSA. The power penalty (left y-axis) is plotted against the input pump power for injection locking $P_{\text {in }}$. The phase noise std causing the penalty is shown on the right y-axis. All the measurements were performed at an optimized slave input power of $-45 \mathrm{dBm}$ as obtained from in Fig. 3(b). At $-50 \mathrm{dBm}$ EDFA input power, the sensitivity degradation is $0.1 \mathrm{~dB}$ due to phase noise, but the presence of the pump would degrade the overall sensitivity by $3 \mathrm{~dB}$ because the signal +idler power is $-50.2 \mathrm{dBm}$. The pump power of $-60 \mathrm{dBm}$ can have a sensitivity degradation of $0.3 \mathrm{~dB}$ due to phase noise and $0.4 \mathrm{~dB}$ due to its presence. The total penalty was $0.7 \mathrm{~dB}$ when locked at $-60 \mathrm{dBm}$ input power and $0.5 \mathrm{~dB}$ when locked at $-65 \mathrm{dBm}$ input power.
In conclusion, we have demonstrated OIL at low powers such as $-65 \mathrm{dBm}$ using a pre-amplifier and a phase locked loop. We identified that the source for the OIL's phase noise is not only the master's phase noise but also the slave laser's linewidth, and this becomes significant at low injection powers. The preamplifier facilitates the reduction of such a phase noise and also allows the reduction of locking power. The pre-amplified OIL set up is employed as a pump regenerator in PSA, and a sensitivity degradation of $0.4 \mathrm{~dB}$ due to the phase noise generated in the OIL is observed for a regenerator input power of $-65 \mathrm{dBm}$.

Funding. Vetenskapsrådet (VR) (2015-00535).

\section{REFERENCES}

1. B. Dahmani, L. Hollberg, and R. Drullinger, Opt. Lett. 12, 876 (1987).

2. Z. Ahmed, H. F. Liu, D. Novak, Y. Ogawa, M. D. Pelusi, and D. Y. Kim, IEEE Photon. Technol. Lett. 8, 37 (1996).

3. C. H. Chang, L. Chrostowski, and C. J. C. Hasnain, IEEE J. Quantum Electron. 9, 1386 (2003).

4. Z. George Pan, S. Jiang, and M. Dagenais, Appl. Phys. Lett. 63, 2999 (1993).

5. Z. Liu, J.-Y. Kim, D. J. Richardson, and R. Slavík, J. Lightwave Technol. 33, 34 (2015).

6. Y. Wang, K. Kasai, M. Yoshida, and M. Nakazawa, Opt. Express 24, 22088 (2016)

7. J. Jignesh, A. Lowery, and B. Corcoran, Opt. Express 26, 5733 (2018).

8. Z. Tong, C. Lundström, P. A. Andrekson, C. J. McKinstrie, M. Karlsson, D. J. Blessing, E. Tipsuwannakul, B. J. Puttnam, H. Toda, and L. Grüner-Nielsen, Nat. Photonics 5, 430 (2011).

9. D. S. Wu, R. Slavik, G. Mari, and D. J. Richardson, J. Lightwave Technol. 31, 2287 (2013)

10. R. Kakarla, K. Vijayan, A. L. Riesgo, and P. A. Andrekson, in Proc. European Conference on Optical Communication (ECOC) (2017), Paper Tu.2.E.3.

11. R. Kakarla, K. Vijayan, J. Schröder, and P. A. Andrekson, in Proc. Optical Fiber Communications Conference and Exposition (OFC) (2018), Paper M4G.2.

12. F. Mogensen, H. Olesen, and G. Jacobsen, IEEE J. Quantum Electron. 21, 784 (1985).

13. J.-Y. Kim, H. Schnatz, D. S. Wu, G. Marra, D. J. Richardson, and R. Slavík, Opt. Lett. 40, 4198 (2015).

14. S. L. I. Olsson, B. Corcoran, C. Lundström, E. Tipsuwannakul, S. Sygletos, A. D. Ellis, Z. Tong, M. Karlsson, and P. A. Andrekson, Opt. Express 21, 14512 (2013).

15. C. Lundström, Z. Tong, M. Karlsson, and P. A. Andrekson, Opt. Lett. 36, 4356 (2011). 